\title{
Non-Gaussian Statistical Interconnect Timing Analysis
}

\author{
Soroush Abbaspour, Hanif Fatemi, Massoud Pedram \\ Department of Electrical Engineering, University of Southern California \\ \{sabbaspo, fatemi, pedram\}@usc.edu
}

\begin{abstract}
This paper focuses on statistical interconnect timing analysis in a parameterized block-based statistical static timing analysis tool. In particular, a new framework for performing timing analysis of $R L C$ networks with step inputs, under both Gaussian and non-Gaussian sources of variation, is presented. In this framework, resistance, inductance, and capacitance of the RLC line are modeled in a canonical first order form and used to produce the corresponding propagation delay and slew (time) in the canonical first-order form. To accomplish this step, mean, variance, and skewness of delay and slew distributions are obtained in an efficient, yet accurate, manner. The proposed framework can be extended to consider higher order terms of the various sources of variation. Experimental results show average errors of less than 2\% for the mean, variance and skewness of interconnect delay and slew while achieving orders of magnitude speedup with respect to a Monte Carlo simulation with $10^{4}$ samples.
\end{abstract}

\section{Introduction}

As the CMOS technology continues to scale down toward Ultra Deep Sub-Micron (UDSM) technologies, variability is becoming a major obstacle to designing high-performance VLSI circuits [1][6]. Due to increased variability, the performance guarantee, and thus, the parametric yield of the designs are being threatened. As a results, there is an urgent need for developing advanced design tools, which are capable of handling variability that stems from imperfect CMOS manufacturing processes (e.g., variations in $\mathrm{L}_{\mathrm{eff}}, \mathrm{T}_{\mathrm{OX}}, \mathrm{V}_{\mathrm{T}}$ or ILD thickness), environmental factors (e.g., drops in $\mathrm{V}_{\mathrm{DD}}$ due to resistive drop and ground bounce and temperature changes due to the creation of local hot spots on the die), or device fatigue phenomena (e.g., electro-migration, hot electron effects, and negative bias temperature instability.) The variability makes it extremely difficult to verify the timing of a design before committing it to manufacturing. Statistical timing analysis (denoted by $\sigma \mathrm{TA}$ ) provides an effective solution to this critical problem [1][3][6][14].

In block-based $\sigma \mathrm{TA}$, every timing quantity of interest (e.g., delay and slew, arrival time and required arrival time) is represented as a function of global sources of variation (denoted by $X_{i}$ ) and independent random sources of variation (denoted by $S_{i}$ ) in the canonical first-order (denoted by CFO) form [6]. As with its STA counterpart, block-based $\sigma \mathrm{TA}$ breaks the analysis task into two parts: 1) statistical interconnect timing analysis and 2) statistical gate timing analysis. Statistical gate timing analysis has been addressed in works such as [4][5][6][7][15]. In this paper, we focus on statistical interconnect timing analysis.

Interconnect timing analysis in STA has been widely studied. AWE methods, which are based on a variable refinement waveform estimator for approximating a generalized linear $R L C$ interconnect, have proven to be quite effective. More precisely, accurate AWE-based interconnect analysis tools (e.g., RICE [9], PRIMA [10]), use the moments of the impulse response to approximate poles of the circuit, and thus, obtain the time-domain waveform of the output under arbitrary inputs. Since more efficient interconnect timing analysis tools are needed during various steps of the physical design flow, there has been a lot of effort to come up with fast interconnect delay metrics and calculators. For instance, Elmore delay uses one moment of the impulse response transfer function to approximate the interconnect delay. The empirical D2M metric [8] uses the first two moments of the impulse response transfer function and propose a closed form delay metric.

For $\sigma \mathrm{TA}$, considering variation of the interconnect, the authors in [2] express the resistance and capacitance of an interconnect line as a linear function of random variables (r.v.'s) and then use these r.v.'s to compute the circuit moments. These variation-aware moments are used in standard closed-form delay metrics such as the Elmore delay metric to compute interconnect delay probability distribution functions (PDF's.) In [3], the authors combine known closedform delay metrics such as Elmore and AWE-based algorithms to take advantage of the efficiency of the first category and the accuracy of the second. Unfortunately, these methods may lead to erroneous results due to the inaccuracy of the closed-form delay metrics. In [12], the authors present a parametric model-order reduction algorithm for addressing the variability of IC interconnect performance. Specifically, they show that variational modeling of interconnects can be cast as a general multi-parameter Krylov-based moment matching problem [13]. They also show that exploiting multi-point expansion under the Krylov framework can lead to compact interconnect models at increased computational cost.

Parameterized block-based $\sigma$ TA tools are still lacking in a practical accurate framework for interconnect timing analysis. In this work, we describe an accurate mathematical framework, which enables one to find the interconnect delay and slew in the CFO from. We point out that, although, in the remainder of this paper, we will mainly focus on the CFO random variables to represent the performance quantities of interest as a function of process and environmental sources of variation, the work itself is not limited to the first-order approximation of these quantities. In fact, it is easy to extend the approach to more complex (e.g., second-order) forms for parameter variations. In addition, although we provide the 
detailed analysis for the step input only, the basic derivations can be extended to the case of a ramped input.

The remainder of this paper is organized as follows. In section 2, we review the background of the parameterized block-based $\sigma \mathrm{TA}$. We also present the technique which converts a variational function into a $\mathrm{CFO}$ form. Interconnect timing analysis for $R L C$ network in block-based $\sigma \mathrm{TA}$ considering step input is presented in section 3. Detailed analysis is presented in section 3.1 while an efficient approximation of statistical interconnect analysis of the solution of section 3.1 is discussed in section 3.2. Experimental results are given in section 4. Conclusions are discussed in section 5. Notice that notations in Table 1 are used throughout this paper.

\section{Background}

In $\sigma \mathrm{TA}$, it is required to evaluate the distribution of the delay and slew of the critical paths. So far, this goal has been done by calculating the mean and variance of the distributions of the delay and slew. However, the sources of variation may exhibit Gaussian or non-Gaussian distributions [14][15] and therefore, results in Gaussian or Non-Gaussian delay distributions. Therefore, in addition to calculating the mean and variance of the timing and electrical parameters, we also calculate the skewness of their distributions.

Table 1: Notation and descriptions

\begin{tabular}{|c|c|}
\hline Notation & Description \\
\hline$h r(t)$ & Impulse response in time domain \\
\hline$H R(s)$ & Impulse response in Laplace domain \\
\hline$s r(t)$ & Step response in time domain \\
\hline$S R(s)$ & Ramp response in time domain \\
\hline$r r(t)$ & Ramp response in Laplace domain \\
\hline$R R(s)$ & $\begin{array}{c}\text { Random variable } A \text { as a function of global and } \\
\text { independent random sources of variations in the } \\
\text { general (non-CFO) form }\end{array}$ \\
\hline$\wp$ & Random variable $A$ in the CFO form \\
\hline$\triangleleft \triangleright$ & \\
\hline$A$
\end{tabular}

Definition: The degree of asymmetry of a distribution is called skewness (denoted by к.) A distribution, or data set, is symmetric if it looks the same to the left and right of the center point. The skewness for a normal distribution is zero. Negative values for the skewness indicate data that are skewed left whereas positive values for the skewness indicate data that are skewed right. By skewed left (right), we mean that the left (right) tail is heavier than the right (left) tail. The skewness of a distribution is defined to be [11]:

$$
\kappa=\frac{\mu_{3}}{\sigma^{3}}
$$

where $\mu_{3}$ is the $3^{\text {rd }}$ central moment and $\sigma^{2}$ is the variance (second central moment.)

Lemma 1: Suppose $\Delta S_{1}, \ldots, \Delta S_{n}$ are $n$ independent random variables with distribution $\Delta S_{i} \sim D i s t_{\mathrm{i}}\left(\mu=0, \sigma^{2}=1, \kappa_{i}\right)$. Then,

$$
\sum_{i=1}^{n} a_{i} \Delta S_{i}=\sqrt{\sum_{i=1}^{n} a_{i}{ }^{2}} \cdot \Delta S_{e q} \quad \text { where } \Delta S_{e q} \sim \text { Dist }\left(\mu=0, \sigma^{2}=1, \kappa=\frac{\sum_{i=1}^{n} a_{i}{ }^{3} \kappa_{i}}{\left(\sum_{i=1}^{n} a_{i}{ }^{2}\right)^{3 / 2}}\right)
$$

Proof: It is omitted for brevity.

\subsection{CFO model in block-based $\sigma \mathrm{TA}$}

In block-based statistical timing analysis tool, a first-order variational model is employed for all timing quantities such as the gate and wire delays, arrival times, required arrival times, slacks and slews, i.e., all timing quantities are expressed in the CFO form as:

$$
\stackrel{\triangleleft}{a}=a_{0}+\sum_{i=1}^{m} a_{i} \Delta X_{i}+a_{m+1} \Delta S_{a}
$$

where $a_{0}$ is the nominal value; $\Delta X_{i}{ }^{\prime} s$ represent the variation of $m$ global sources of variation, $X_{i}$, from their nominal values, $a_{i}$ 's are the sensitivities to each of the global sources of variation, $\Delta S_{a}$ is the variation of independent random variable $S_{a}$ and $a_{m+1}$ is the sensitivity of the timing quantity to $S_{a}$. By scaling the sensitivity coefficients, we can assume that $\Delta X_{i}$ and $\Delta S_{a}$ have distributions with $\mu=0$ and $\sigma^{2}=1$ and skewness $=\kappa$ denoted by $\operatorname{Dist}\left(\mu=0, \sigma^{2}=1, \kappa\right)$.

Variation in the physical dimensions of the wire causes change in its resistance and capacitance, thereby, making the gate delay and slew as well as wire delay and slew to vary accordingly. Therefore, we need to capture the effect of geometric variations on the electrical parameters. Classifying the sources of variation into global and independent random sources of variation, we represent electrical parameters of the wire (i.e., resistance and capacitance) in the CFO form. For instance, resistance and capacitance in the $\mathrm{CFO}$ form are calculated as follows:

$$
\stackrel{\triangleleft}{r}=r_{0}+\sum_{i=1}^{m} r_{i} \Delta X_{i}+r_{m+1} \Delta S_{r} ; \stackrel{\triangleleft}{c}=c_{0}+\sum_{i=1}^{m} c_{i} \Delta X_{i}+c_{m+1} \Delta S_{c}
$$

where $r_{0}$ and $c_{0}$ represent nominal resistance and capacitance values, computed when the wire dimensions are at their nominal or typical values. $\Delta X_{i}$ 's are the global sources of variation and $\Delta S_{r}$ and $\Delta S_{c}$ represent the independent random sources of variation for the resistance and capacitance, respectively; $r_{i}$ and $c_{i}$ are the sensitivity coefficients of resistance and capacitance with respect to the sources of variations, respectively. With appropriate scaling of the sensitivity coefficients, we can assume that $\Delta X_{i}, \Delta S_{r}$, and $\Delta S_{c}$ have distributions with $\mu=0$ and $\sigma^{2}=1$ and skewness $=\kappa$ denoted by $\operatorname{Dist}\left(\mu=0, \sigma^{2}=1, \kappa\right)$.

Observation: Invariant Functional Form Property: This property states that: $y=f(x) \Leftrightarrow \stackrel{\mathscr{Q}}{Y}=f(\stackrel{\mathscr{\wp}}{X})$, which follows from the fact that the form of function $f$ is independent of its input type (i.e., whether it is deterministic or variational.)

\subsection{Conversion to CFO form}

As mentioned earlier, it is important to represent timing and electrical quantities in the CFO form. This in turn enables one to propagate first order sensitivities to different sources of variation through the circuit timing graph [7]. In addition, it makes statistical computation efficient and practical and provides timing diagnostics at the cost of a small cost in run time. The remaining question is how to convert a quantity of interest (which itself is a function of different $\mathrm{CFO}$ variables) into the $\mathrm{CFO}$ form. 
In general, suppose that $G$ is a nonlinear function of $m$ global and $p$ independent random sources of variation. To represent $G$ in the CFO form, we differentiate the function with respect to the global and independent random sources of variation, and therefore it can be written as:

$$
\begin{aligned}
& \stackrel{\wp}{G}\left(\Delta X_{1} \ldots \Delta X_{m}, \Delta S_{1} \ldots \Delta S_{p}\right) \quad \stackrel{\wp}{G} \text { is a non-CFO function of } \\
& \text { sources of variations }
\end{aligned}
$$

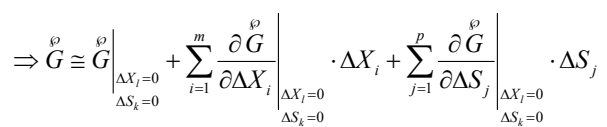

$$
\begin{aligned}
& =\left.\stackrel{\wp}{G}\right|_{\substack{\Delta X_{i}=0 \\
\Delta S_{k}=0}}+\left.\sum_{i=1}^{m} \frac{\partial G}{\partial \Delta X_{i}}\right|_{\substack{\Delta X_{i}=0 \\
\Delta S_{k}=0}} \cdot \Delta X_{i}+\left.\sum_{j=1}^{p} \sqrt{\left(\frac{\partial G}{\partial \Delta S_{j}}\right)^{2}}\right|_{\substack{\Delta X_{i}=0 \\
\Delta S_{k}=0}} \cdot \Delta S_{G} \\
& =\stackrel{\triangleleft}{b} \quad \text { where }\left\{\begin{array}{l}
l=1 \ldots m \\
k=1 \ldots p
\end{array}\right.
\end{aligned}
$$

where the distribution of $\Delta S_{G}$ can be calculated using Lemma 1.

\section{Statistical Interconnect Timing Analysis}

Problem Statement: Given is an $R L C$ network representation where every electrical parameter $(r, l$, and $c)$ is in the CFO form. Considering a step input, the objective is to calculate the minimum mean squared error (MSE) fit of the distribution of the $\alpha \%$ transition time at any node in the network in the CFO form such that:

$$
E\left\{\left[\left\{\begin{array}{l}
\wp(\Delta \triangleright \\
t_{\alpha}
\end{array}\right)-\alpha\right]^{2}\right\} \text { is minimized }
$$

where $t_{\alpha}$ is the $\alpha \%$ transition time and $v(t)$ is the voltage of the node in the time domain.

\subsection{Detailed Analysis}

In STA, we have seen that for a general $R L C$ circuit, the impulse response transfer function from the input node to any node of the circuit can be written as:

$$
H R(s)=\frac{V_{x}(s)}{V_{\text {in }}(s)}=\sum_{i=0}^{u} a_{i} s^{i} / \sum_{j=0}^{v} b_{j} s^{j}
$$

where $a_{i}$ and $b_{j}$ can be represented as functions of the electrical parameters of the circuit. If a unit step input voltage is applied to the circuit, the voltage step response at any node of the circuit in Laplace domain may be written as;

$$
V_{x}(s)=S R(s)=\frac{H R(s)}{s}
$$

In $\sigma \mathrm{TA}$, due to the variability of the electrical parameters, $a_{i}$ 's and $b_{j}{ }^{\text {'s }}$ are not deterministic scalar values. Instead, by using the technique in section 2.2, we represent them in the $\mathrm{CFO}$ form. Thus, we can rewrite the revised impulse response in Laplace domain as:

$$
\stackrel{\wp}{H R(s)}=\frac{\stackrel{\wp}{V}_{x}(s)}{V_{\text {in }}(s)}=\sum_{i=0}^{u} a_{i}^{\triangleleft \triangleright} s^{i} / \sum_{j=0}^{v} b_{j} s^{j}
$$

$$
\text { where }\left\{\begin{array}{l}
\triangleleft \triangleright \\
a_{i}=a_{i, 0}+\sum_{k=1}^{m} a_{i, k} \Delta X_{k}+\sum_{l=1}^{p} a_{i, m+l}^{*} \Delta S_{l} \\
\triangleleft \triangleright \\
b_{j}=b_{j, 0}+\sum_{k=1}^{m} b_{j, k} \Delta X_{k}+\sum_{l=1}^{p} b_{j, m+l}^{*} \Delta S_{l}
\end{array}\right.
$$

where $a_{i, 0}$ and $b_{j, 0}$ are the nominal values of the $s^{i}$ and $s^{j}$ coefficient, respectively; $\Delta X_{k}, k=1 \ldots . m$ represent all $m$ global sources of variation; $\Delta S_{l}, l=1 \ldots p$ are all independent random sources of variation in the circuit; $a_{i, k}$ is the sensitivity of $a_{i}$ to $\Delta X_{k} ; b_{j, k}$ is the sensitivity of $b_{j}$ to $\Delta X_{k} ; a_{i, m+l}^{*}$ is the sensitivity of $a_{i}$ to $\Delta S_{l}$; and $b_{j, m+l}^{*}$ is the sensitivity of $b_{j}$ to $\Delta S_{l}$. For instance, if a $s^{i}$ coefficient is not a function of the either global or independent random sources of variation, then the corresponding sensitivity coefficient will be zero.

We are interested in calculating the interconnect delay and slew in the CFO form. Assuming a step input is applied to the $R L C$ network, the following theorem shows how to evaluate $\alpha \%$ transition time of voltage step response of any node in the network in its CFO form. From this result, we can easily find the solution to the problem statement.

Theorem: Consider an $R L C$ network excited by a step input. Assume that each electrical parameter $(r, l$, and $c)$ of the tree is in the CFO form. The MSE fit of the distribution of the $\alpha \%$ transition time of any node in the network in the CFO form is calculated as follows:

$$
\stackrel{\Delta \triangleright}{t_{\alpha}=t_{\alpha, 0}}+\sum_{i=1}^{m} t_{\alpha, i} \Delta X_{i}+\sum_{j=1}^{p}{ }_{t_{\alpha, m+j}}^{*} \Delta S_{j}=t_{\alpha, 0}+\sum_{i=1}^{m} t_{\alpha, i} \Delta X_{i}+t_{\alpha, m+1} \Delta S_{t_{\alpha}}
$$

where

$$
t_{\alpha, 0}=v_{x}^{-1}(\alpha)=s r^{-1}(\alpha)
$$

$$
t_{\alpha, i}=\frac{-\Lambda_{i}\left(t_{\alpha, 0}\right)}{h r\left(t_{\alpha, 0}\right)} ; \quad \Lambda_{i}(t)=L^{-1}\left\{\left.\frac{\partial}{\partial \Delta X_{i}}\left(\frac{\left.\begin{array}{r}
\wp \\
H R(s)
\end{array}\right)}{s}\right)\right|_{\begin{array}{l}
\Delta X_{i}=0 \\
\Delta S_{k}=0
\end{array}}\right\}
$$

$$
\begin{aligned}
& t_{\alpha, m+1}=\sqrt{\left(t_{\alpha, m+1}^{*}\right)^{2}+\ldots+\left(t_{\alpha, m+p}^{*}\right)^{2}} ; \\
& t_{\alpha, m+j}^{*}=\frac{-\Psi_{m+j}\left(t_{\alpha, 0}\right)}{h r\left(t_{\alpha, 0}\right)} ; \quad \Psi_{m+j}(t)=L^{-1}\left\{\left.\frac{\partial}{\partial \Delta S_{j}}\left(\frac{H R(s)}{s}\right)\right|_{\substack{\Delta X_{l}=0 \\
\Delta S_{k}=0}}\right\}
\end{aligned}
$$

The distribution of $\Delta S_{t_{\alpha}}$ is given as:

$$
\Delta S_{t_{\alpha}} \sim \operatorname{Dist}\left(\mu=0, \sigma^{2}=1, \kappa=\sum_{j=1}^{p} t_{\alpha, m+j}^{*}{ }^{3} \kappa_{j} /\left(\sum_{i=1}^{n} t_{\alpha, m+j}^{*}{ }^{2}\right)^{3 / 2}\right)
$$

Proof: See appendix.

Eqn. (5) (which is simply the inverted form of $v_{x}\left(t_{\alpha, 0}\right)=\alpha$ ) means that to calculate $t_{\alpha, 0}$ (i.e., the nominal value of the $\alpha \%$ transition time), we must perform delay analysis for a step input with all the circuit elements set to their respective nominal values, which can be done by using any timing calculator such as AWE methods [17]. $h r\left(t_{\alpha, 0}\right)$ in Eqn's (6) and (7) is the impulse response of the circuit at time $t_{\alpha, 0}$ when the circuit is in its nominal condition, and can be calculated by AWE methods [17]. This value appears in all of the coefficients and is independent from the variations, so it should be calculated only once. 
As shown in Eqn. (6), $\Lambda_{i}\left(t_{\alpha, 0}\right)$ is equal to the inverse Laplace of the "derivative of non-CFO step response" when sources of variations are set to zero. Therefore, if we substitute the non-CFO impulse response (cf. Eqn. (3)) into Eqn. (6), then $\Lambda_{i}\left(t_{\alpha, 0}\right)$ may be calculated as a function of the $a_{i, 0}$ and $b_{j, 0}$, and sensitivity coefficients of $a_{i, l}, b_{j, k}, a^{*}{ }_{i, m+l}$ ,$b_{j, m+l}^{*}$. These coefficients are calculated by using the RICE program [9]. Similar set of steps are performed to calculate $\Psi_{m+j}(t)$. Finally, Eqn. (8) provides the skewness of $\Delta S_{t_{\alpha}}$.

Figure 1 presents the summary of the algorithm for statistical timing analysis, called SITA.

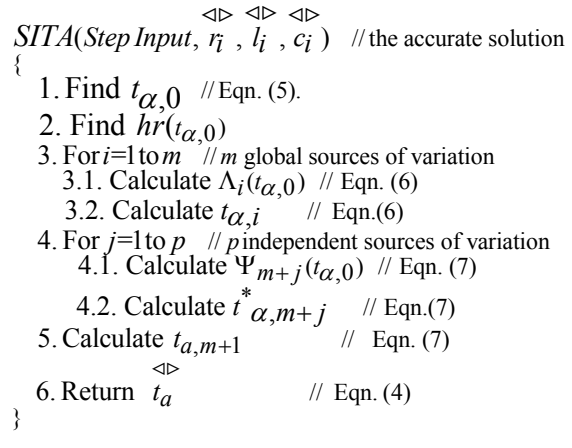

Figure 1: General SITA Algorithm

\subsection{An Approximate Solution}

Eqn's (4)-(8) return an accurate solution of the parameterized block-based non-Gaussian statistical timing analysis in the CFO form. However, it is useful to have an efficient, yet reasonably accurate, solution for interconnect timing analysis during the design flow. Therefore, we propose the following approximate algorithm.

The CPU-intensive portion of the SITA algorithm is calculating $h r\left(t_{\alpha, 0}\right), \Lambda_{i}\left(t_{\alpha, 0}\right)$, and $\Psi_{m+j}\left(t_{\alpha, 0}\right)$. Thus, we use exponential waveform approximation to calculate $h r\left(t_{\alpha, 0}\right)$ and single-pole approximation to calculate $\Lambda_{i}\left(t_{\alpha, 0}\right)$ and $\Psi_{m+j}\left(t_{\alpha, 0}\right)$. These approximations will result in the following equations (The derivations are omitted for readability):

$$
\begin{array}{r}
t_{\alpha, i} \cong \frac{k\left(1-e^{-\omega t_{\alpha, 0}}\right) t_{\alpha, 0}}{(1-\alpha) \ln (1-\alpha)^{-1}} \text { where } k=\frac{a_{0,0} b_{0, i}-a_{0, i} b_{0,0}}{b_{0,0}{ }^{2}} \\
\omega=\frac{b_{0,0}}{2 b_{1,0}-b_{0,0}\left(\frac{a_{1, i} b_{0,0}+a_{0, i} b_{1,0}-a_{1,0} b_{0, i}-a_{0,0} b_{1, i}}{a_{0, i} b_{0,0}-a_{0,0} b_{0, i}}\right)} \\
t_{\alpha, m+j}^{*}=\frac{k^{*}\left(1-e^{-\omega^{*} t_{\alpha, 0}}\right) t_{t} \alpha, 0}{(1-\alpha) \ln (1-\alpha)^{-1}} \text { where } k^{*}=\frac{a_{0,0} b_{0, m+j}-a_{0, m+j} b_{0,0}}{b_{0,0}{ }^{2}} \\
\omega^{*}=\frac{b_{0,0}}{2 b_{1,0}-b_{0,0}\left(\frac{a_{1, m+j} b_{0,0}+a_{0, m+j} b_{1,0}-a_{1,0} b_{0, m+j}-a_{0,0} b_{1, m+j}}{a_{0, m+j} b_{0,0}-a_{0,0} b_{0, m+j}}\right)}
\end{array}
$$

The proposed approximate solution to the general SITA algorithm is presented in Figure 2, called SITA-Aprx.

\section{Experimental Results}

We used two different circuit configurations for generating the experimental results. The first one consists of an $R L C$ ladder topology with 5 segments connected in series (nodes are labeled 1 to 5 from the input to the output) as shown in
Figure 3(a), whereas the second one is an $R C$ tree with two branches as depicted in Figure 3(b). A step input is applied to both interconnect configurations.

$$
\begin{aligned}
& \text { SITA_Aprx (Step Input, } \left.r_{i}, l_{i}, c_{i}\right) \quad / / \text { An approximate solution } \\
& \text { 1. Find } t_{\alpha, 0} \\
& \text { // Use delay metrics such as Elmore, D2M, ... for finding } 0-\alpha \% \\
& \text { transition of the node for the nominal condition of the circuit. } \\
& \text { 2. For } i=1 \text { to } m / / m \text { global sources of variation } \\
& \text { 2.1. Calculate } t_{\alpha, i} / / \text { Eqn. (9) } \\
& \text { 3. For } j=1 \text { to } p / / p \text { independent sources of variation } \\
& \text { 3.1. Calculate } t^{*} \alpha, m+j \quad / / \text { Eqn.(10) } \\
& \text { 4. Calculate } t_{\alpha, m+1} \quad / / \text { Eqn. (7) } \\
& \text { 5. Return } t_{\alpha} \quad / / \text { Eqn. (4) }
\end{aligned}
$$

Figure 2: SITA-Aprx Algorithm

We considered three different global sources of variation (interconnect width, interconnect thickness, and the ILD variation) and one independent random source of variation for each electrical parameter (i.e., $r, l$, and $c$ ) in the circuit. We used empirical capacitance modeling equations [16] to compute linear coefficients (sensitivities) of the CFO model. We considered a 3-sigma variation of $30 \%$ for all sources of variation. We also assumed that sources of variation are skewed with different skewness values as explained in each subsection. The mean, variance, and skewness of interconnect delay and also 10\%-90\% transition times (slew) at a number of nodes (highlighted in Figure 3), were calculated by using both SITA and SITA-Aprx algorithms (In SITA-Aprx algorithm, we used $D 2 M$ delay metric for calculating $\left.t_{\alpha, 0}\right)$. We also performed Monte Carlo simulation with 10,000 samples for each test scenario and recorded the resulting mean, variance, and skewness of interconnect delay and 10\%-90\% transition times. Average percentage errors between results obtained by the Monte Carlo (Actual) and results based on SITA and SITA-Aprx algorithms (Analytical) are reported.

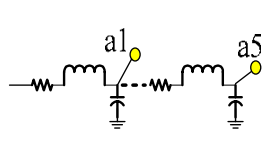

(a)

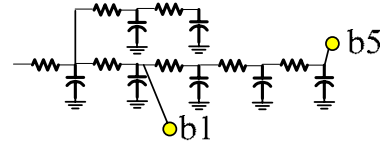

(b)
Figure 3. (a) RLC ladder and (b) RC tree.

\section{A. RLC ladder with 5 series-connected segments}

The load in this section is considered to be an $R L C$ ladder with 5 segments. The nominal values of Width, Thickness, and ILD are chosen to be $(W)^{\text {nom }}=\{0.4,0.5,0.6,0.7,0.8\} \mu \mathrm{m}$, $(T)^{\text {nom }}=\{0.4,0.5,0.6,0.7,0.8\} \mu \mathrm{m},(I L D)^{n o m}=\{0.2,0.3,0.4$, $0.5,0.6\} \mu \mathrm{m}$. The scaled distribution of the sources of variation is considered to have a skewness of $0.4,0.6$, and 0.8 . We performed our experiments by using both algorithms SITA and SITA-Aprx - as explained before. The results are shown in Table 2 for the skewness of 0.4 , in Table 3 for the skewness of 0.6 , and in Table 4 for the skewness of 0.8 . Experimental results show an average error of $1 \%, 2 \%$, and $2 \%$ for mean, variance and skewness of interconnect delay and slew for SITA algorithm and an average error of $4 \%, 3 \%$, and $3 \%$ for mean, variance and skewness of interconnect delay and slew for the SITA-Aprx algorithm. The error in 
node " $a_{1}$ " is more than the error in node " $a_{5}$ " when the SITAAprx is utilized. This is due to the resistive shielding effect. However, when using SITA, error levels in both nodes are close to one another. As the skewness value increases from 0.4 to 0.8 , the error in the calculated mean, variance, and skewness of delay and slew increases, but only slightly.

The only source of error when using the SITA algorithm is the linear first order modeling (CFO equations) for the timing and electrical parameters. For SITA-Aprx, the delay metric for calculating $t_{\alpha, 0}$, the single-pole approximation of $\Lambda$ and $\Psi$ and the method of impulse response calculation presented in section 3.2, increases the error (fortunately by a small amount.) However, the runtime of the SITA algorithm is on average 90 times shorter than that of the Monte Carlo based approach. Furthermore, the runtime of SITA-Aprx is on average about 15 times shorter than the SITA runtime.

Table 2: Average error (\%) for the $R L C$ ladder (Skewness=0.4)

\begin{tabular}{|c|c|c|c|c|c|c|c|c|}
\hline & \multicolumn{4}{|c|}{ Delay } & \multicolumn{4}{c|}{ Slew } \\
\hline \multirow{2}{*}{ Average error } & \multicolumn{2}{|c|}{ SITA } & \multicolumn{2}{c|}{ SITA-Aprx } & \multicolumn{2}{c|}{ SITA } & \multicolumn{2}{c|}{ SITA-Aprx } \\
\cline { 2 - 9 } & $a_{1}$ & $a_{5}$ & $a_{1}$ & $a_{5}$ & $a_{1}$ & $a_{5}$ & $a_{1}$ & $a_{5}$ \\
\hline Mean & 0.6 & 0.5 & 3.9 & 3.0 & 0.7 & 0.7 & 3.6 & 3.4 \\
\hline Variance & 1.2 & 1.1 & 2.7 & 2.2 & 1.4 & 1.0 & 2.6 & 1.9 \\
\hline Skewness & 1.6 & 1.8 & 3.5 & 3.1 & 2.3 & 2.2 & 3.0 & 2.7 \\
\hline
\end{tabular}

Table 3: Average error (\%) for the $R L C$ ladder (Skewness=0.6)

\begin{tabular}{|c|c|c|c|c|c|c|c|c|}
\hline & \multicolumn{4}{|c|}{ Delay } & \multicolumn{4}{c|}{ Slew } \\
\hline \multirow{2}{*}{ Average error } & \multicolumn{2}{|c|}{ SITA } & \multicolumn{2}{c|}{ SITA-Aprx } & \multicolumn{2}{c|}{ SITA } & \multicolumn{2}{c|}{ SITA-Aprx } \\
\cline { 2 - 10 } & $a_{1}$ & $a_{5}$ & $a_{1}$ & $a_{5}$ & $a_{1}$ & $a_{5}$ & $a_{1}$ & $a_{5}$ \\
\hline Mean & 0.9 & 1.3 & 4.7 & 3.1 & 1.0 & 0.9 & 4.3 & 3.7 \\
\hline Variance & 1.5 & 1.8 & 3.3 & 2.5 & 1.7 & 1.5 & 3.1 & 2.4 \\
\hline Skewness & 2.0 & 2.1 & 3.8 & 3.2 & 2.2 & 2.0 & 3.4 & 2.9 \\
\hline
\end{tabular}

Table 4: Average error (\%) for the $R L C$ ladder (Skewness=0.8)

\begin{tabular}{|c|c|c|c|c|c|c|c|c|}
\hline & \multicolumn{4}{|c|}{ Delay } & \multicolumn{4}{c|}{ Slew } \\
\hline \multirow{2}{*}{ Average error } & \multicolumn{2}{|c|}{ SITA } & \multicolumn{2}{|c|}{ SITA-Aprx } & \multicolumn{2}{c|}{ SITA } & \multicolumn{2}{c|}{ SITA-Aprx } \\
\cline { 2 - 10 } & $a_{1}$ & $a_{5}$ & $a_{1}$ & $a_{5}$ & $a_{1}$ & $a_{5}$ & $a_{1}$ & $a_{5}$ \\
\hline Mean & 1.1 & 1.4 & 4.9 & 4.0 & 1.2 & 1.4 & 4.5 & 4.1 \\
\hline Variance & 1.9 & 2.1 & 3.8 & 3.3 & 2.1 & 1.9 & 3.7 & 2.9 \\
\hline Skewness & 2.6 & 2.2 & 3.5 & 2.9 & 2.2 & 2.0 & 3.3 & 3.2 \\
\hline
\end{tabular}

\section{B. $R C$ tree with two branches}

Now the interconnect topology is an $R C$ tree as shown in Figure 3(b). The nominal value of Width, Thickness, and ILD are again chosen to be $(W)^{n o m}=\{0.4,0.5,0.6,0.7,0.8\} \mu \mathrm{m}$, $(T)^{n o m}=\{0.4,0.5,0.6,0.7,0.8\} \mu \mathrm{m},(I L D)^{n o m}=\{0.2,0.3,0.4$, $0.5,0.6\} \mu \mathrm{m}$. The scaled distribution of the sources of variation is considered to have a skewness of $0.5,0.75$, and 1 .

We performed the experiments by employing both SITA and SITA-Aprx algorithms. Results are reported in Table 5 for the skewness of 0.5 , Table 6 for the skewness of 0.75 , and Table 7 for the skewness of 1 . Experimental results show an average error of $1 \%, 3 \%$, and $2 \%$ for mean, variance and skewness of interconnect delay and slew for SITA algorithm and an average error of $5 \%, 3 \%$, and $3 \%$ for mean, variance and skewness of interconnect delay and slew for the SITAAprx algorithm. As the skewness value increases from 0.5 to 1 , error in the calculated mean, variance, and skewness of delay and slew increases slightly. Similar observations apply with respect to the sources of error in SITA and SITA-Aprx algorithms. Finally, note that SITA is on average 94 times faster than the Monte Carlo based approach while SITA-Aprx is on average 18 times faster than SITA.

\section{Conclusion}

We presented an analytical framework for handling the nonGaussian statistical interconnect timing analysis in blockbased statistical static timing analysis. We expressed the resistance and capacitance of a line in canonical first order forms. We subsequently utilized these forms to perform accurate timing analysis of $R L C$ interconnect, thereby, producing the CFO form of the propagation delay and slew of interconnects. Experimental results showed an average error of $1 \%, 2 \%$, and $2 \%$ for mean, variance and skewness of interconnect delay and slew for SITA algorithm and a speed up of two orders of magnitude compared to Monte Carlo simulation results with $10^{4}$ samples. Furthermore we proposed an approximate analytical method to solve the aforesaid problem. The average error of this approximation is about $5 \%$, $3 \%$, and $3 \%$ for mean, variance and skewness of interconnect delay and slew compared to Monte Carlo simulation while offering a speed up of at least one order of magnitude compared to the accurate analytical method.

Table 5: Average error (\%) for the $R C$ tree (Skewness=0.5)

\begin{tabular}{|c|c|c|c|c|c|c|c|c|}
\hline & \multicolumn{4}{|c|}{ Delay } & \multicolumn{4}{c|}{ Slew } \\
\hline \multirow{2}{*}{ Average error } & \multicolumn{2}{|c|}{ SITA } & \multicolumn{2}{|c|}{ SITA-Aprx } & \multicolumn{3}{|c|}{ SITA } & \multicolumn{2}{c|}{ SITA-Aprx } \\
\cline { 2 - 9 } & $b_{1}$ & $b_{5}$ & $b_{1}$ & $b_{5}$ & $b_{1}$ & $b_{5}$ & $b_{1}$ & $b_{5}$ \\
\hline Mean & 0.5 & 0.8 & 4.9 & 4.0 & 0.8 & 0.9 & 4.2 & 3.3 \\
\hline Variance & 2.8 & 2.5 & 3.3 & 3.1 & 2.5 & 2.3 & 3.2 & 3.3 \\
\hline Skewness & 2.2 & 2.1 & 2.8 & 2.7 & 3.1 & 3.0 & 3.1 & 3.1 \\
\hline
\end{tabular}

Table 6: Average error (\%) for the $R C$ tree (Skewness=0.75)

\begin{tabular}{|c|c|c|c|c|c|c|c|c|}
\hline \multirow{3}{*}{ Average error } & \multicolumn{4}{|c|}{ Delay } & \multicolumn{4}{|c|}{ Slew } \\
\hline & \multicolumn{2}{|c|}{ SITA } & \multicolumn{2}{|c|}{ SITA-Aprx } & \multicolumn{2}{|c|}{ SITA } & \multicolumn{2}{|c|}{ SITA-Aprx } \\
\hline & $b_{1}$ & $b_{5}$ & $b_{1}$ & $b_{5}$ & $b_{1}$ & $b_{5}$ & $b_{1}$ & $b_{5}$ \\
\hline Mean & 0.9 & 1.0 & 5.4 & 5.1 & 1.1 & 1.2 & 5.0 & 4.3 \\
\hline Variance & 3.0 & 2.7 & 3.2 & 3.4 & 3.2 & 3.1 & 3.2 & 3.1 \\
\hline Skewness & 2.5 & 2.3 & 3.2 & 3.0 & 2.5 & 2.4 & 3.2 & 3.4 \\
\hline \multicolumn{9}{|c|}{ Table 7: Average error (\%) for the $R C$ tree (Skewness=1) } \\
\hline & \multicolumn{4}{|c|}{ Delay } & \multicolumn{4}{|c|}{ Slew } \\
\hline \multirow{2}{*}{ Average error } & \multicolumn{2}{|c|}{ SITA } & \multicolumn{2}{|c|}{ SITA-Aprx } & \multicolumn{2}{|c|}{ SITA } & \multicolumn{2}{|c|}{ SITA-Aprx } \\
\hline & $b_{1}$ & $b_{5}$ & $b_{1}$ & $b_{5}$ & $b_{1}$ & $b_{5}$ & $b_{1}$ & $b_{5}$ \\
\hline Mean & 1.3 & 1.2 & 5.8 & 5.2 & 1.4 & 1.3 & 6.0 & 5.1 \\
\hline Variance & 3.2 & 3.1 & 3.5 & 3.1 & 3.4 & 2.9 & 3.9 & 3.1 \\
\hline Skewness & 2.7 & 2.6 & 3.9 & 3.2 & 2.4 & 2.6 & 3.7 & 3.1 \\
\hline
\end{tabular}

\section{References}

[1] R. Nassif, "Modeling and Analysis of Manufacturing Variations," CICC, pp. 223-228, 2001.

[2] K. Agarwal, D. Sylvester, D. Blaauw, F. Liu, S. Nassif, S. Vrudhula, "Variational delay metrics for interconnect timing analysis," DAC, Proceedings. 41st, June 7-11, 2004 Pages:381 - 384, 2004.

[3] S. Abbaspour, H. Fatemi, and M. Pedram, "VITA: Variation-aware interconnect timing analysis for symmetric and skewed sources of variation considering variational ramp input," Proc. of Great Lakes Symposium on VLSI, Apr. 2005, pp. 426-430.

[4] Agarwal, A.; Dartu, F.; Blaauw, D.;"Statistical gate delay model considering multiple input switching", Design Automation Conference, 2004. Proceedings. 41st, June 7-11, 2004 Pages:658 - 663

[5] Okada, K.; Yamaoka, K.; Onodera, H.; "A statistical gate-delay model considering intra-gate variability" Computer Aided Design, 2003. ICCAD-2003. 9-13 Nov. 2003 Pages:908 - 913

[6] S. Abbaspour, H. Fatemi, and M. Pedram, "Parameterized block-based non-Gaussian statistical gate timing analysis," To appear in Proc. of Asia and South Pacific Design Automation Conference, Jan. 2006.

[7] Visweswariah, C.; Ravindran, K.; Kalafala, K.; Walker, S.G.; Narayan, S.;'First-order incremental block-based statistical timing analysis", DAC, 2004. Proceedings. 41st , June 7-11, 2004, Pages:331 - 336 
[8] C. Alpert, A. Devgan, and C. Kashyap, "RC delay metrics for performance optimization," IEEE Trans. on Computer Aided Design, vol. 20, pp. 571-582. 2001.

[9] C. L. Ratzlaff, N. Gopal, and L. T. Pillage, "RICE: rapid interconnect circuit evaluator," Proc. of 28th ACM/IEEE Design Automation Conference, pp. 555-560, 1991

[10] A. Odabasioglu, M. Celik, and L. T. Pileggi, "PRIMA: passive reducedorder interconnect macromodeling algorithm," Proceedings of ICCAD San Jose, pp. $58-65,1997$.

[11] http://www.mathworld.com

[12] P. Li, F. Liu, X. Li, L. T. Pileggi, and S. R. Nassif, "Modeling Interconnect Variability Using Efficient Parametric Model Order Reduction" Design, Automation and Test in Europe (DATE'05).

[13] L. Daniel, O. Siong, L. Chay, K. Lee and J. White, "A multi-parameter moment-matching model-reduction approach for generating geometrically parameterized interconnect performance models," IEEE Trans. CAD, vol. 23, no. 5, pp 678-693, May , 2004.

[14] X. Li, J. Le, P. Gopalakrishnan, and L. T. Pileggi, "Asymptotic Probability Extraction for Non-Normal Distributions of Circuit Performance," ICCAD, pp 2-9, November 2004.

[15] H. Chang, V. Zolotov, C. Visweswariah, and S. Narayan, "Parameterized block-based statistical timing analysis with nonGaussian and nonlinear parameters", DAC, pp. 71-76, June 2005.

[16] J. Chern, J. Huang, L. Arledge, P. Li, and P. Yang, "Multilevel Metal Capacitance Models for CAD Design Synthesis Systems," IEEE Electron Devices Letters, pp. 32-34, Jan. 1992.

[17] L. T. Pillage, R. A. Rohrer," Asymptotic waveform evaluation for timing analysis", IEEE Trans. Computer-Aided Design of Integrated Circuits and Systems, pp. 352-366, Apr. 1990.

\section{Appendix}

Definition: The inverse of the Laplace transform can be calculated as:

$$
F(t)=\frac{1}{2 \pi i} \int_{\gamma-i \infty}^{\gamma+i \infty} f(s) \cdot e^{s t} d s
$$

where $\gamma$ is a vertical contour in the complex plane chosen so that all singularities of $f(s)$ are to the left of it [11]

Proof of Theorem: Since $v\left(t_{\alpha}\right)$ is a random variable, we must find $t_{\alpha}$ in the CFO form s.t.

$$
\left.E\left\{\left[\begin{array}{l}
\wp \\
t_{\alpha}
\end{array}\right)-\alpha\right]^{2}\right\} \text { is minimized }
$$

The above nonlinear stochastic equation is not easy to evaluate, therefore, we approximate $v\left(t_{\alpha}\right)$ with

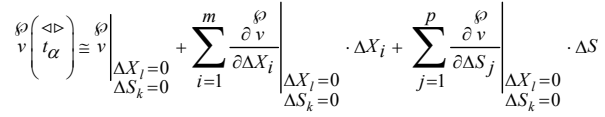

$$
\begin{aligned}
& \left\{\begin{array}{l}
l=1 \ldots . . m \\
k=1 \ldots p
\end{array}\right.
\end{aligned}
$$

To satisfy the minimization in Eqn. (12), we have;

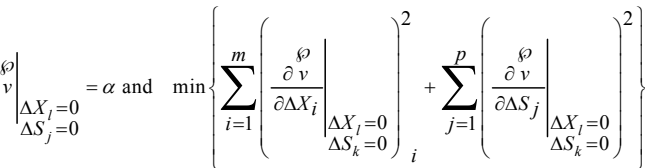

$$
\begin{aligned}
& \left.\Rightarrow \frac{\partial v}{\partial \Delta X_{i}}\right|_{\substack{\Delta X_{l}=0 \\
\Delta S_{k}=0}}=0 \quad \forall i=1 \ldots . . m ;\left.\quad \frac{\partial v}{\partial \Delta S_{j}}\right|_{\substack{\Delta X_{l}=0 \\
\Delta S_{k}=0}}=0 \quad \forall j=1 \ldots . p
\end{aligned}
$$

From the definition, the first condition in Eqn. (14) can be written as:

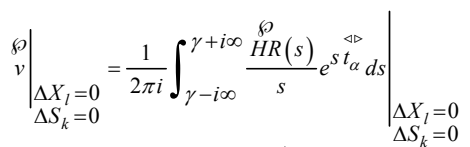

$$
\begin{aligned}
& =\frac{1}{2 \pi i} \int_{\gamma-i \infty}^{\gamma+i \infty} \frac{\left.\stackrel{\wp}{H R(s)}\right|_{\Delta X_{l}=0}}{s} e^{s S_{\alpha, 0}} d s=\alpha
\end{aligned}
$$

Therefore,

$$
\begin{gathered}
v_{x}\left(t_{\alpha, 0}\right)=s r\left(t_{\alpha, 0}\right)=\frac{1}{2 \pi i} \int_{\gamma-i \infty}^{\gamma+i \infty} \frac{H R^{\mathrm{nom}}(s)}{s} e^{-s t_{\alpha, 0}} d s=\alpha \\
\text { where } H R^{\mathrm{nom}}(s)=\frac{\sum_{i=0}^{u} a_{i, 0} s^{i}}{\sum_{j=0}^{v} a_{j, 0} s^{j}}
\end{gathered}
$$

Thus, $t_{\alpha, 0}=s r^{-1}(\alpha)$; which proves Eqn (5). The second condition in Eqn. (14) may be treated as follows:

$$
\begin{aligned}
\left.\frac{\partial v}{\partial \Delta X_{i}}\right|_{\substack{\Delta X_{l}=0 \\
\Delta S_{k}=0}}=0 \quad \forall i=\left.1 \ldots m \Rightarrow \frac{\partial}{\partial \Delta X_{i}}\left(\frac{1}{2 \pi i} \int_{\gamma-i \infty}^{\gamma+i \infty} \frac{H R(s)}{s} e^{s t_{\alpha}} d s\right)\right|_{\substack{\Delta X_{l}=0 \\
\Delta S_{k}=0}} \\
\left.=\frac{1}{2 \pi i} \int_{\gamma-i \infty}^{\gamma+i \infty} \frac{\frac{\partial}{\partial \Delta X i}(H R(s)}{\wp}\right)\left.\right|_{\substack{\Delta X_{l}=0 \\
\Delta S_{k}=0}} e^{s t_{\alpha, 0} d s} \\
+\left.t_{\alpha, i} \cdot \frac{1}{2 \pi i} \int_{\gamma-i \infty}^{\gamma+i \infty}\left(\begin{array}{c}
\wp \\
H R(s)
\end{array}\right)\right|_{\substack{\Delta X_{l}=0 \\
\Delta S_{k}=0}} e^{s t_{\alpha, 0} d s=0}
\end{aligned}
$$

Now,

$$
\begin{aligned}
& \Lambda(t)=L^{-1}\left\{\left.\frac{\partial}{\partial \Delta X_{i}}\left(\begin{array}{c}
\wp \\
H R(s)
\end{array}\right)\right|_{\substack{\Delta X_{l}=0 \\
\Delta S_{k}=0}}\right\} \\
& =L^{-1}\left\{\frac{\sum_{l=1}^{u} a_{l, i} l \sum^{l} \sum_{k=1}^{v} b_{k, 0} s^{k}-\sum_{l=1}^{u} a_{l, 0} s^{l} \sum_{k=1}^{v} b_{k, p} s^{k}}{s\left(\sum_{k=1}^{v} b_{k, 0} s^{k}\right)^{2}}\right\}
\end{aligned}
$$

which proves Eqn (6). Finally, to prove Eqn. (7) we can write;

$$
\begin{aligned}
& \left.\frac{\partial v}{\partial \Delta S_{j}}\right|_{\substack{\Delta X_{l}=0 \\
\Delta S_{k}=0}}=0 \quad \forall j=\left.1 \ldots p \Rightarrow \frac{\partial}{\partial \Delta S_{j}}\left(\frac{1}{2 \pi i} \int_{\gamma-i \infty}^{\gamma+i \infty} \frac{H R(s)}{s} e^{-s t_{\alpha}} d s\right)\right|_{\substack{\Delta X_{l}=0 \\
\Delta S_{k}=0}} \\
& =\frac{1}{2 \pi i} \int_{\gamma-i \infty}^{\gamma+i \infty} \frac{\left.\frac{\partial}{\partial \Delta S_{j}}\left(\begin{array}{c}
\wp \\
H R(s)
\end{array}\right)\right|_{\substack{\Delta X_{l}=0 \\
\Delta S_{k}=0}} e^{-s t_{\alpha, 0} d s}}{s} \\
& +t_{\alpha, m+j}^{*} \cdot \frac{1}{2 \pi i} \int_{\gamma-i \infty}^{\gamma+i \infty} H R^{n o m}(s) \cdot e^{-s t_{\alpha, 0}} d s=0
\end{aligned}
$$

Consequently,

$$
\begin{gathered}
\Psi(t)=L^{-1}\left\{\frac{\partial}{\partial \Delta S_{j}}\left(\begin{array}{c}
\wp R(s) \\
H R
\end{array}\right) \mid \begin{array}{c}
\Delta X_{l}=0 \\
\Delta S_{k}=0
\end{array}\right\} \\
=L^{-1}\left\{\frac{\sum_{l=1}^{u} a_{k, m+j} s^{s} \sum_{k=1}^{v} b_{k, 0^{s}}{ }^{k}-\sum_{l=1}^{u} a_{l, 0} s^{l} \sum_{k=1}^{v} b_{k, m+j} s^{k}}{\left(\sum_{k=1}^{v} b_{k, 0} s^{k}\right)^{2}}\right\} \\
\Rightarrow t_{\alpha, m+j}^{*}=\frac{-\Psi\left(t_{\alpha, 0}\right)}{h r\left(t_{\alpha, 0}\right)}
\end{gathered}
$$

